\title{
Treating elevated LDL cholesterol in patients with low short-term risk: Decision making at the limits of EBM
}

\section{Mark J Pletcher ${ }^{1,2}$}

10.1136/ebmed-2015-110257

${ }^{1}$ Department of Epidemiology and Biostatistics, University of California, San Francisco,

California, USA

${ }^{2}$ Division of General Internal

Medicine, Department of

Medicine, University of

California, San Francisco,

California, USA

Correspondence to:

Dr Mark J Pletcher,

Department of Epidemiology and Biostatistics, University of California, San Francisco 185 Berry Street, Suite 5700, San Francisco, CA 94107, USA; mpletcher@epi.ucsf.edu

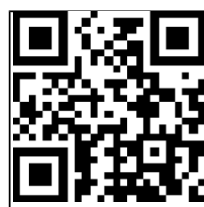

\section{SLinked}

- http://dx.doi.org/10.1136/ ebmed-2015-110170
Cholesterol and statins are among the most extensively researched topics in clinical medicine, but controversy continues to rage over how to interpret this vast and growing body of evidence and translate it into better clinical care for patients. In 2013, after a 9-year hiatus, guidelines for treatment of high cholesterol were updated by the American College of Cardiology and the American Heart Association. ${ }^{1}$ The new guidelines shifted treatment recommendations in several important ways, perhaps the most important of which was to recommend that statin primary prevention treatment decisions be made nearly entirely on the basis of a patient's overall risk for atherosclerotic cardiovascular disease instead of accounting for the low-density lipoprotein cholesterol (LDL) level (unless LDL is extremely elevated). This increasing emphasis on risk-based statin prescribing strikingly demotes LDL levels in importance compared with previous guidelines, shifts prescribing towards older men who are at higher average short-term risk, ${ }^{2}$ and has elicited controversy. ${ }^{3}$

In this issue of $E B M$, Sauser and colleagues review the evidence for LDL-lowering treatment of persons with an elevated level of LDL cholesterol but relatively low short-term (10-year) cardiovascular risk. ${ }^{4}$ The basic rationale for the 'early LDL treatment hypothesis' is that high-LDL cholesterol causes cumulative damage to coronary arteries (in the form of atherosclerosis) even during young adulthood when risk of cardiovascular events is low, and that early LDL-lowering could reduce that damage accumulation and thereby reduce coronary heart disease risk in the long term more than waiting to treat LDL cholesterol until later in life when event risk becomes higher. To assess the evidence for this early LDL treatment hypothesis, the authors conducted an extensive review of randomised controlled trials of LDL-lowering and patient outcomes and longitudinal observational studies including Mendelian randomisation studies, and solicited additional specific arguments and sources of evidence from established experts.

After excluding arguments based on pathophysiology, the authors identified three fundamental arguments supporting the early LDL treatment hypothesis and focused their evidence review accordingly. These arguments are: (1) that patients at lower cardiovascular risk get more benefit per amount of LDL reduction; (2) that statin trials demonstrate a 'legacy effect' whereby cardiovascular risk of treated patients continues to be reduced even after the trial ends; and (3) that Mendelian randomisation studies demonstrate that lifelong LDL-lowering from genetic factors produces a much larger relative risk reduction than seen in statin trials. The authors found support for arguments 1 and 3, no support for 2 (though they did not consider an oft-cited legacy effect trial $^{5}$ ), provide methodological critiques and alternative explanations for the supporting evidence, and point out a critical gap in the evidence: that there is no clinical study (certainly no randomised controlled trial) demonstrating that LDL-lowering with a statin early in life, when cardiovascular risk is low, is actually superior in terms of reducing clinical events than waiting to start statin therapy until cardiovascular risk is elevated (eg, 10-year risk $>7.5 \%$ ).

This last point is irrefutably true. The only direct way to demonstrate that early LDL treatment would reduce cardiovascular events more than 'late' treatment (waiting until risk is elevated) would be to randomly assign patients with elevated LDL but low risk to immediate versus delayed (risk-based) statin therapy, and then follow those persons through many decades of life to see if cardiovascular events are reduced later in life by early treatment. The length of follow-up alone (essential to the design) and inevitable resulting challenges with engagement and compliance over that long follow-up in the context of a rapidly changing landscape (new drugs, new ways to predict risk), as well as the likely immense cost of such a study, make it quite unlikely that any such trial will ever be conducted. One reasonable answer to the question posed by this review, therefore, is something of a forgone conclusion: that there is "no clinical evidence for or against whether starting before 10-year risk is $7.5-12.5 \%$ provides substantial additional net patient benefit'.

In the absence of that direct clinical evidence, we must do our best to glean indirect evidence to inform this critical clinical question. Sauser and colleagues add substantially to this debate by their creative reframing of arguments, careful literature review and intelligent discussion of flaws in the attempts of others to use indirect evidence to support the early LDL treatment hypothesis. These flaws should certainly mitigate the enthusiasm of early LDL treatment hypothesis advocates.

It is unclear, however, why pathophysiological arguments, which were excluded from consideration by the authors, should be irrelevant to this evidence review. Atherosclerosis is the unquestioned primary intermediary between cardiovascular risk factors such as LDL cholesterol and clinical cardiovascular events (though there are others, such as inflammation and thrombosis). Large and well-designed observational studies have demonstrated clear associations between cardiovascular risk factors and atherosclerosis early in life, ${ }^{6-9}$ persistence of atherosclerosis from early in life into later life, ${ }^{10}{ }^{11}$ and between atherosclerosis and clinical events ${ }^{12}$; and a randomised trial has shown that statin treatment early in life reduces atherosclerosis. ${ }^{13}$ Recent evidence, cited in this review, demonstrates that cumulative exposure to hyperlipidaemia during young adulthood is an independent risk factor for cardiovascular events later in life. ${ }^{14}$ While this evidence also has flaws (some pointed out by Sauser et al), it supports the 
pathophysiological argument and indirect evidence for early LDL treatment.

Additional evidence has recently accumulated on another related aspect of the guidelines-whether to treat to an LDL target. Recent randomised controlled trials demonstrate that newer non-statin medications, when used in conjunction with statins to drastically reduce LDL levels, appear to reduce clinical events in high-risk patients. ${ }^{15-17}$ The decision to initiate LDL-lowering therapy with a statin in a low-risk patient is not the same as the decision to intensify LDL-lowering treatment in a high-risk patient, and this new evidence does not mean ezetimibe and PCSK9 inhibitors should have a place in current guidelines, but it does seem to add weight to the argument that LDL levels matter. ${ }^{18}$

Comparing cholesterol guidelines to blood pressure guidelines provides an interesting perspective. As for cholesterol, only indirect evidence supports lowering blood pressure during early adulthood when overall cardiovascular risk is low. However, in spite of similarities in the evidence profile, blood pressure guidelines clearly recommend treatment based on a high level of the risk factor without consideration for cardiovascular risk. ${ }^{19}$ There are undeniable differences in the evidence base for these two risk factors, but the clear discrepancy in this specific 'early treatment' aspect of the recommendations raises questions about whether historical considerations or other non-evidence-based factors may be influencing the decisions of these parallel guideline committees as they interpret the evidence base.

As experts continue to debate the strength of the indirect evidence supporting the early LDL treatment hypothesis, clinicians must make real-world decisions about treating their patients with elevated LDL cholesterol and low short-term risk. Just as the guideline authors hedged their bets by recommending statins when LDL is over $190 \mathrm{mg} / \mathrm{dL}$, clinicians can hedge their bets for patients by taking degree of LDL elevation into account, and there is little evidence supporting the rather arbitrary $190 \mathrm{mg} / \mathrm{dL}$ threshold. As clinicians decide how high is too high for any particular patient, they are well-justified in using all possible sources of information to make their best guess about what is right for their patients. This should clearly include patient preference about statin use, as antipathy to taking a pill every day and concerns about adverse effects can easily overwhelm the small average absolute benefits of statin therapy. ${ }^{20}$ It might include weighing the cost of statin therapy obtainable by the patient, ${ }^{20}$ though such utilitarian reasoning can feel repugnant to clinicians and patients. It might even make sense to perform additional testing for atherosclerosis, such as with CT-enabled quantification of coronary calcium, ${ }^{21}$ or genetic markers of risk for cardiovascular disease or statin effectiveness. ${ }^{22}$ All things considered (and we must consider all things when making decisions at the limits of $E B M$ ), the decision to start statins in the context of high cholesterol and low short-term risk remains a difficult one for patients and clinicians. Though the definitive trial may not be feasible, anything we can do to generate more indirect evidence, such as opening access to statin trial data currently maintained by the Cholesterol Treatment Trialists group to more researchers (as called for by
Sauser and colleagues) and generating additional knowledge relevant to the decision (such as long-term statin safety data) will certainly be welcome.

Competing interests None declared.

Provenance and peer review Commissioned; internally peer reviewed.

\section{References}

1. Stone NJ, Robinson J, Lichtenstein AH, et al. 2013 ACC/AHA guideline on the treatment of blood cholesterol to reduce atherosclerotic cardiovascular risk in adults: a report of the American College of Cardiology/American Heart Association Task Force on Practice Guidelines. Circulation, 2013.

2. Pencina MJ, Navar-Boggan AM, D'Agostino RB Sr, et al. Application of new cholesterol guidelines to a population-based sample. $N$ Engl J Med 2014;370:1422-31.

3. Ridker PM, Wilson PW. A trial-based approach to statin guidelines. JAMA 2013;310:1123-4.

4. Sauser K, Levine DA, Hayward RA. A Review of the clinical evidence related to early treatment of elevated LDL for cardiovascular primary prevention. Evidence based medicine 2015;20:162-9.

5. Ford I, Murray H, Packard CJ, et al. Long-term follow-up of the West of Scotland Coronary Prevention Study. $N$ Engl J Med 2007;357:1477-86

6. Berenson GS, Srinivasan SR, Bao W, et al. Association between multiple cardiovascular risk factors and atherosclerosis in children and young adults. The Bogalusa Heart Study. $N$ Engl J Med 1998;338:1650-6.

7. Mahoney LT, Burns TL, Stanford W, et al. Coronary risk factors measured in childhood and young adult life are associated with coronary artery calcificaction in young adults: the Muscatine Study. J Am Coll Cardiol 1996;27:277-84.

8. Raitakari OT, Juonala M, Kahonen M, et al. Cardiovascular risk factors in childhood and carotid artery intima-media thickness in adulthood: the Cardiovascular Risk in Young Finns Study. JAMA 2003;290:2277-83.

9. [No authors listed]. Relationship of atherosclerosis in young men to serum lipoprotein cholesterol concentrations and smoking. A preliminary report from the Pathobiological Determinants of Atherosclerosis in Youth (PDAY) Research Group. JAMA 1990;264:3018-24.

10. Davis PH, Dawson JD, Riley WA, et al. Carotid intimal-medial thickness is related to cardiovascular risk factors measured from childhood through middle age: the Muscatine Study. Circulation 2001;104:2815-9.

11. Pletcher MJ, Bibbins-Domingo K, Liu K, et al. Non-optimal lipids commonly present in young adults and coronary calcium later in life: the CARDIA (Coronary Artery Risk Development in Young Adults) Study. Ann Intern Med 2010;153:137-46.

12. Detrano R, Guerci AD, Carr JJ, et al. Coronary calcium as a predictor of coronary events in four racial or ethnic groups. $N$ Engl J Med 2008;358:1336-45.

13. Wiegman A, Hutten BA, de Groot E, et al. Efficacy and safety of statin therapy in children with familial hypercholesterolemia: a randomized controlled trial. JAMA 2004;292:331-7.

14. Navar-Boggan AM, Peterson ED, D'Agostino RB Sr, et al. Hyperlipidemia in early adulthood increases long-term risk of coronary heart disease. Circulation 2015;131: 451-8.

15. Cannon CP, Blazing MA, Giugliano RP, et al. Ezetimibe added to statin therapy after acute coronary syndromes. $N$ Engl J Med 2015;372:2387-97.

16. Robinson JG, Farnier M, Krempf M, et al. Efficacy and safety of alirocumab in reducing lipids and cardiovascular events. $N$ Engl J Med 2015;372:1489-99. 
17. Sabatine MS, Giugliano RP, Wiviott SD, et al. Efficacy and safety of evolocumab in reducing lipids and cardiovascular events. N Engl J Med 2015;372:1500-9.

18. Jarcho JA, Keaney JF Jr. Proof that lower is better-LDL cholesterol and IMPROVE-IT. $N$ Engl J Med 2015;372: 2448-50.

19. Go AS, Bauman MA, Coleman King SM, et al. An effective approach to high blood pressure control: a science advisory from the American Heart Association, the American College of Cardiology, and the Centers for Disease Control and Prevention. Hypertension 2014;63:878-85.
20. Pandya A, Sy S, Cho S, et al. Cost-effectiveness of 10-year risk thresholds for initiation of statin therapy for primary prevention of cardiovascular disease. JAMA 2015;314:142-50.

21. Pletcher MJ, Pignone M, Earnshaw S, et al. Using the coronary artery calcium score to guide statin therapy: a cost-effectiveness analysis. Circ Cardiovasc Qual Outcomes 2014;7:276-84.

22. Mega JL, Stitziel NO, Smith JG, et al. Genetic risk, coronary heart disease events, and the clinical benefit of statin therapy: an analysis of primary and secondary prevention trials. Lancet 2015;385:2264-71. 